\title{
The pedicled anterolateral thigh flap for trochanteric pressure sore reconstruction: Technical notes to optimize surgical outcomes
}

\author{
Mahmoud A. Hifny \\ Department of Plastic Surgery, Qena University Hospital, Faculty of Medicine, South Valley University, Qena, Egypt
}

\begin{abstract}
Background The pedicled anterolateral thigh (ALT) flap has become more popular for the reconstruction of soft-tissue defects in neighboring areas. Nonetheless, few studies in the literature have explored the use of this flap for trochanteric ulcer reconstruction. The aim of our study is to present the author's experience of utilizing the ALT flap, with a focus on technical elements regarding the flap design and the tunneling method to maximize the reach of the flap.

Methods The medical records of patients who received pedicled ALT flaps for the reconstruction of trochanteric pressure sores were retrospectively reviewed. The patients' demographic data, operative details, and postoperative complications were evaluated.

Results Between October 2018 and December 2019, 10 consecutive patients (age range, 1345 years) underwent 11 pedicled ALT myocutaneous flaps for trochanteric pressure sore reconstruction. Each flap was designed around the most distal cutaneous perforator that was included in the proximal third of the skin paddle. The flaps ranged in size from $11 \times 6$ to $14 \times 8 \mathrm{~cm}$. The ALT flap was transposed through a lateral subcutaneous tunnel in five patients, while the open tunnel technique was used in six patients. All flaps survived, and no vascular compromise was observed.

Conclusions The pedicled ALT flap is a safe and reliable option for reconstructing trochanteric pressure sores. An appropriate flap design and a good choice of the tunneling method are crucial for successful flap transposition.
\end{abstract}

Keywords Anterolateral thigh flap / Trochanteric pressure sores / Reconstruction
Correspondence: Mahmoud A. Hifny Department of Plastic Surgery, Qena University Hospital, Faculty of Medicine, South Valley University, Qena 83523, Egypt Tel: $+20-1019700881$ Fax: +20-963211279 E-mail: mahmoud_abdallah0211@ med.svu.edu.eg

Received: June 27, $2020 \bullet$ Revised: October 20, $2020 \bullet$ Accepted: October 27, 2020

pISSN: 2234-6163 • elSSN: 2234-6171 • https://doi.org/10.5999/aps.2020.01270 • Arch Plast Surg 2021;48:114-120

\section{INTRODUCTION}

Trochanteric pressure sores frequently occur as a complication in long-term bedridden patients, especially in those with substantial flexion contracture who recline in the lateral position. The surgical management of trochanteric pressure sores requires proper flap selection; in particular, the flap should offer well-vascularized tissue with significant muscle bulk to obliterate the ulcer dead space, as well as providing easy transposition for tension-free closure [1].

Although the tensor fascia lata (TFL) flap remains the workhorse flap for trochanteric region reconstruction due to its reliability and ease of harvest [2], it has significant shortcomings. In recent years, the pedicled anterolateral thigh (ALT) flap has be- 
come more popular, and it now has an established role in reconstruction of defects in the perineum, groin, anterior abdominal wall, and thigh [3-7]. However, few case series in the literature have reported utilizing the pedicled ALT flap for trochanteric pressure sore reconstruction [8-11].

The aim of our study is to present the author's experiences of employing the ALT myocutaneous flap as a proximally-based pedicle island flap for the reconstruction of trochanteric pressure sores, focusing on the technical aspects of flap design and the tunneling method in order to maximize the flap's reach while providing a safe flap transfer.

\section{METHODS}

\section{Study design}

A retrospective review was conducted of the medical records of patients who underwent reconstruction of trochanteric pressure sores using pedicled ALT flaps between October 2018 and December 2019 at the plastic surgery department of our institution. This study was performed with permission from the Institutional Review Board of the Qena Faculty of Medicine (IRB No. SVU-MED-PLS-0134-201087) and in accordance with the ethical standards that laid down in the 1964 Declaration of Helsinki. Written informed consent was obtained from each patient. The patients' demographic characteristics, defect size, flap dimensions, tunneling technique for flap transposition, flap complications, donor site morbidity, and follow-up time were evaluated (Table 1).

\section{Operative technique}

All procedures were performed with the patient in the supine position and under either general or spinal anesthesia. Surgical treatment consisted of radical debridement with resection of all devitalized soft tissues and ulcer bursae, followed by smoothly trimming the bony prominences of the greater trochanter (Figs. $1 \mathrm{~A}, 3 \mathrm{~A})$. Bacteriological samples from the soft tissues and bone were obtained in all cases. The longitudinal axis of the ALT flap was designed along a line passing from the anterior superior iliac spine to the lateral border of the patella. The vascular perforators that were detected by Doppler ultrasonography were usually located within a $3-\mathrm{cm}$ circle on the mid-point of this line. After the identification of all perforators, it was necessary to select the most reliable distal skin perforator to obtain the longest pedicle possible and to maximize the arc of flap rotation. The desired skin island was marked around the selected distal perforator, which should be placed eccentrically in the proximal third of the flap (Figs. 1B, 2A, 3B).

The medial border of the flap was incised through the deep fascia over the rectus femoris muscles until identification of the yellow fatty intermuscular septum between the rectus femoris and vastus lateralis muscles distally. Once identified, the septum between both muscles was opened from the distal to proximal aspects, and the rectus femoris muscle was then displaced medially away from the vastus lateralis muscle to identify the descending branch of the lateral circumflex femoral artery, along with its associated perforators [12].

After identification of the previously selected distal perforators, the desired size of the skin paddle, with a considerable part of the vastus lateralis muscle, was then incised and dissected quickly because intramuscular dissection of the perforators is not necessary (Fig. 2B). The quantity of muscle harvested from the vastus lateralis should be equal to the width of the skin paddle to ensure good filling of the ulcer dead space.

Next, the vascular pedicle was dissected to the origin of the descending branch of the lateral circumflex femoral artery (Fig. 2B). taking care to avoid injuring the ascending branch of the lateral circumflex femoris artery to preserve the TFL flap for reconstruction if trochanteric pressure sores recurred [9].

\section{Table 1. Patients data and operative results}

\begin{tabular}{|c|c|c|c|c|c|c|}
\hline Case No. & Age $(y r) / s e x$ & Ulcer size $(\mathrm{cm})$ & Flap size $(\mathrm{cm})$ & Tunneling method & Complication & Follow-up time (mon) \\
\hline 1 & $23 / \mathrm{M}$ & $10 \times 6$ & $12 \times 7$ & Subcutaneous & None & 14 \\
\hline 2 & $21 / M$ & $12 \times 6$ & $13 \times 7$ & Subcutaneous & None & 12 \\
\hline 3 & $40 / F$ & $13 \times 5$ & $14 \times 6$ & Open & None & 11 \\
\hline 4 & $35 / \mathrm{M}$ & $13 \times 6$ & $14 \times 7$ & Open & None & 9 \\
\hline 5 & $45 / M$ & $12 \times 7$ & $13 \times 8$ & Open & Wound dehiscence & 7 \\
\hline 6 & $45 / \mathrm{M}$ & $13 \times 7$ & $14 \times 8$ & Open & None & 6 \\
\hline 7 & $13 / \mathrm{M}$ & $10 \times 6$ & $12 \times 7$ & Subcutaneous & None & 5 \\
\hline 8 & $42 / F$ & $10 \times 5$ & $11 \times 6$ & Open & None & 4 \\
\hline 9 & $25 / \mathrm{M}$ & $12 \times 6$ & $13 \times 7$ & Subcutaneous & None & 3 \\
\hline 10 & 29/M & $11 \times 6$ & $13 \times 7$ & Subcutaneous & None & 3 \\
\hline 11 & $37 / M$ & $11 \times 7$ & $13 \times 8$ & Open & None & 2 \\
\hline
\end{tabular}

M, male; F, female. 


\section{Fig. 1. ALT flap with the subcutaneous tunnel technique}

(A) Preoperative appearance of a left trochanteric pressure sore. (B) Design of a pedicled anterolateral thigh (ALT) myocutaneous flap measuring $12 \times 7 \mathrm{~cm}$; the flap skin paddle was designed around the most distal perforators, which were located in the proximal third of the flap. (C) Passage of the ALT flap using the subcutaneous tunnel technique. (D) Immediate postoperative view of the ALT flap.
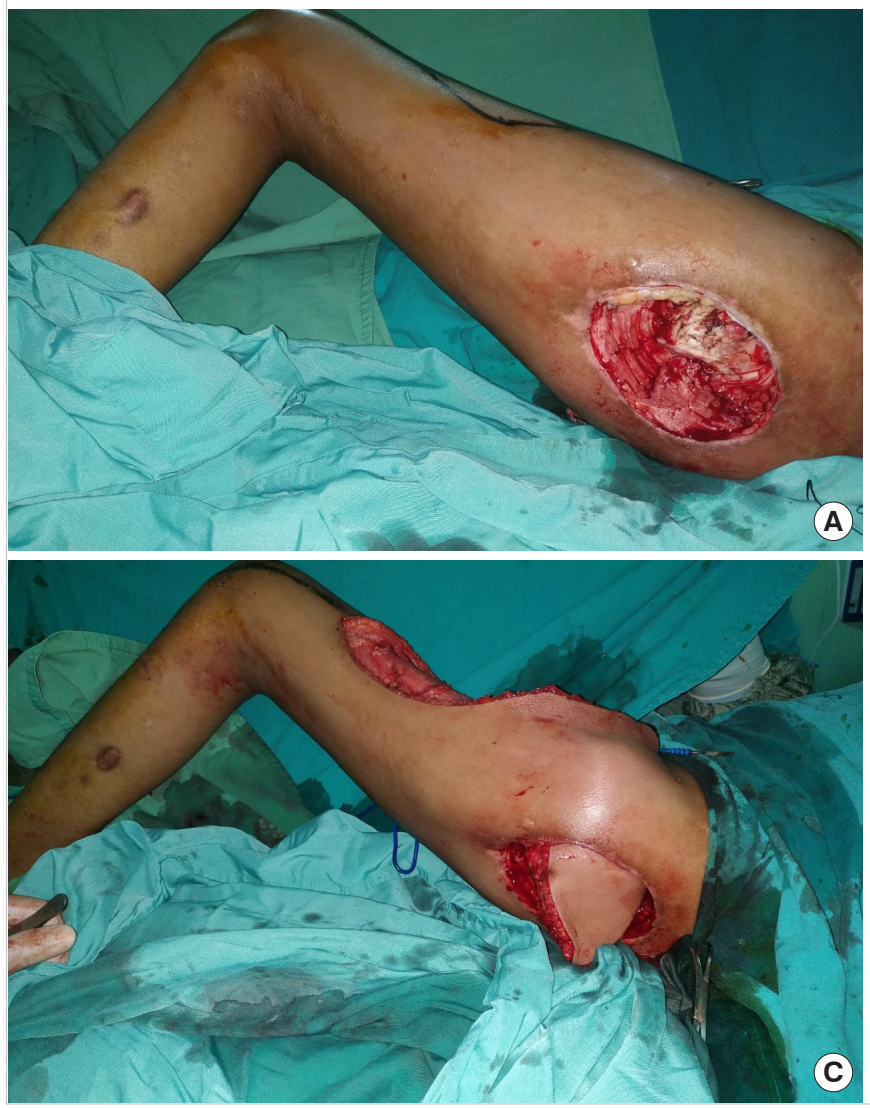

Upon harvesting of the flap, the pedicled ALT flap was transposed into the trochanteric defect either via a subcutaneous tunnel in the lateral thigh (Fig. 1C) or through an open tunnel created by dividing the skin bridge between the defect and the flap (Figs. 2C, 3C). Myotomy in the vastus lateralis muscle was done to make a trough to inset the flap vascular pedicle. Primary closure of the donor site was performed after drain insertion (Figs. 1D, 2C, 3D). The postoperative protocol involved a 4-week period of strict avoidance of the lateral decubitus position at the operative site. The appropriate type of antibiotic therapy and duration of treatment were guided by wound cultures, and patients with positive bone cultures received intravenous antibiotics for 6 weeks to treat osteomyelitis.

\section{RESULTS}

Between October 2018 and December 2019, 10 consecutive patients (eight men and two women) underwent 11 pedicled ALT myocutaneous flaps for trochanteric pressure sore reconstruction. Their mean age was 32.2 years (range, 13-45 years).
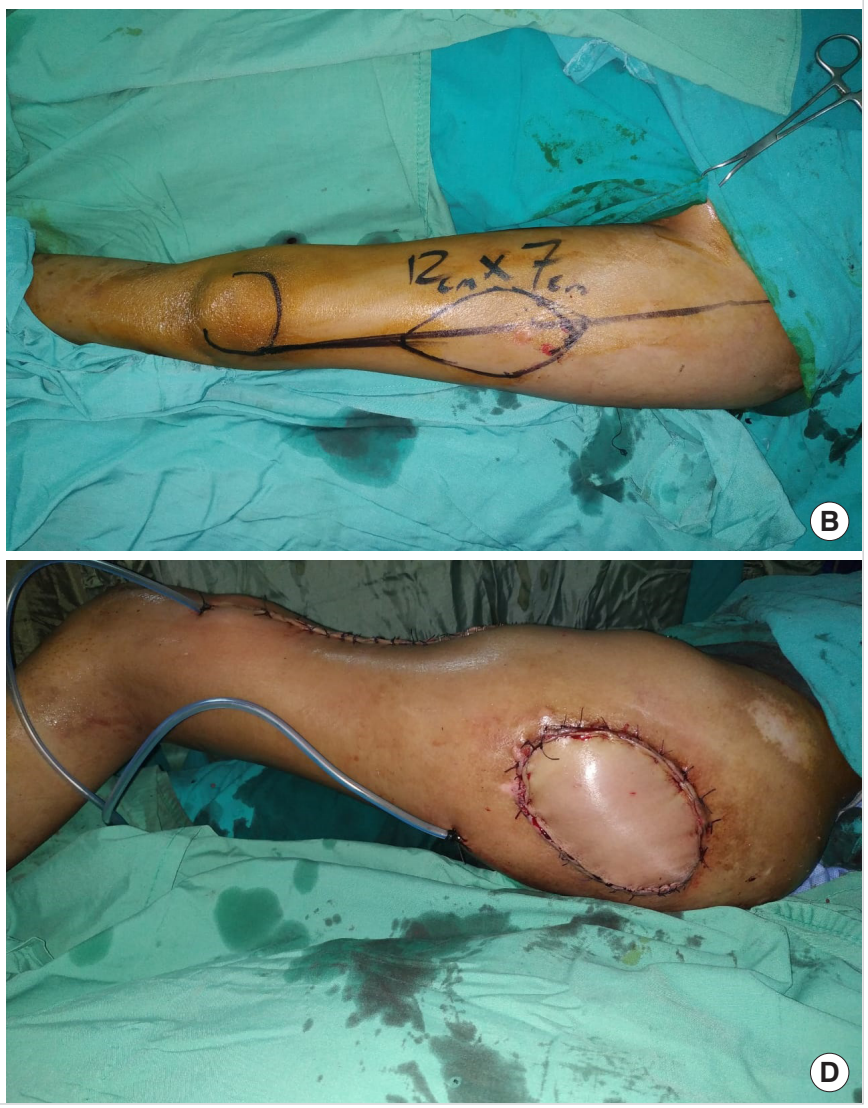

All patients were paraplegic as a result of previous spinal cord injuries. The flaps ranged in size from $11 \times 6 \mathrm{~cm}\left(66 \mathrm{~cm}^{2}\right)$ to $14 \times 8 \mathrm{~cm}\left(122 \mathrm{~cm}^{2}\right)$. The desired skin island was marked around the most distal cutaneous perforator that was detected. There were one or two distal perforators, which should be placed eccentrically in the proximal third of the flap. All these perforators were musculocutaneous, and were identified and transferred together with the vastus lateralis muscle without skeletonization. All flaps were harvested as myocutaneous flaps. According to the method of flap transposition, we categorized our patients into two groups. In the first group $(n=5)$, the ALT myocutaneous flap was transposed through a lateral subcutaneous tunnel to reach the recipient. In the second group $(n=6)$, the open tunnel technique was used, with division of the skin bridge between the recipient and donor sites. All flaps survived without any vascular compromise or postoperative complications, and satisfactory coverage was achieved in all cases. All donor-site wounds were closed primarily and healed uneventfully without any complications, except for one case of small wound dehiscence that was managed by secondary stitches. The aver- 


\section{Fig. 2. ALT flap with the open tunnel technique}

(A) Preoperative appearance of a left trochanteric pressure sore with the design of a pedicled anterolateral thigh (ALT) myocutaneous flap measuring $13 \times 8 \mathrm{~cm}$; the flap skin paddle was designed around the most distal perforator, which lay in the proximal third of the flap. (B) Elevation of the ALT myocutaneous flap. (C) Immediate postoperative results of the ALT flap after passage of the flap using the open tunnel technique. (D) Postoperative photo after 3 months.
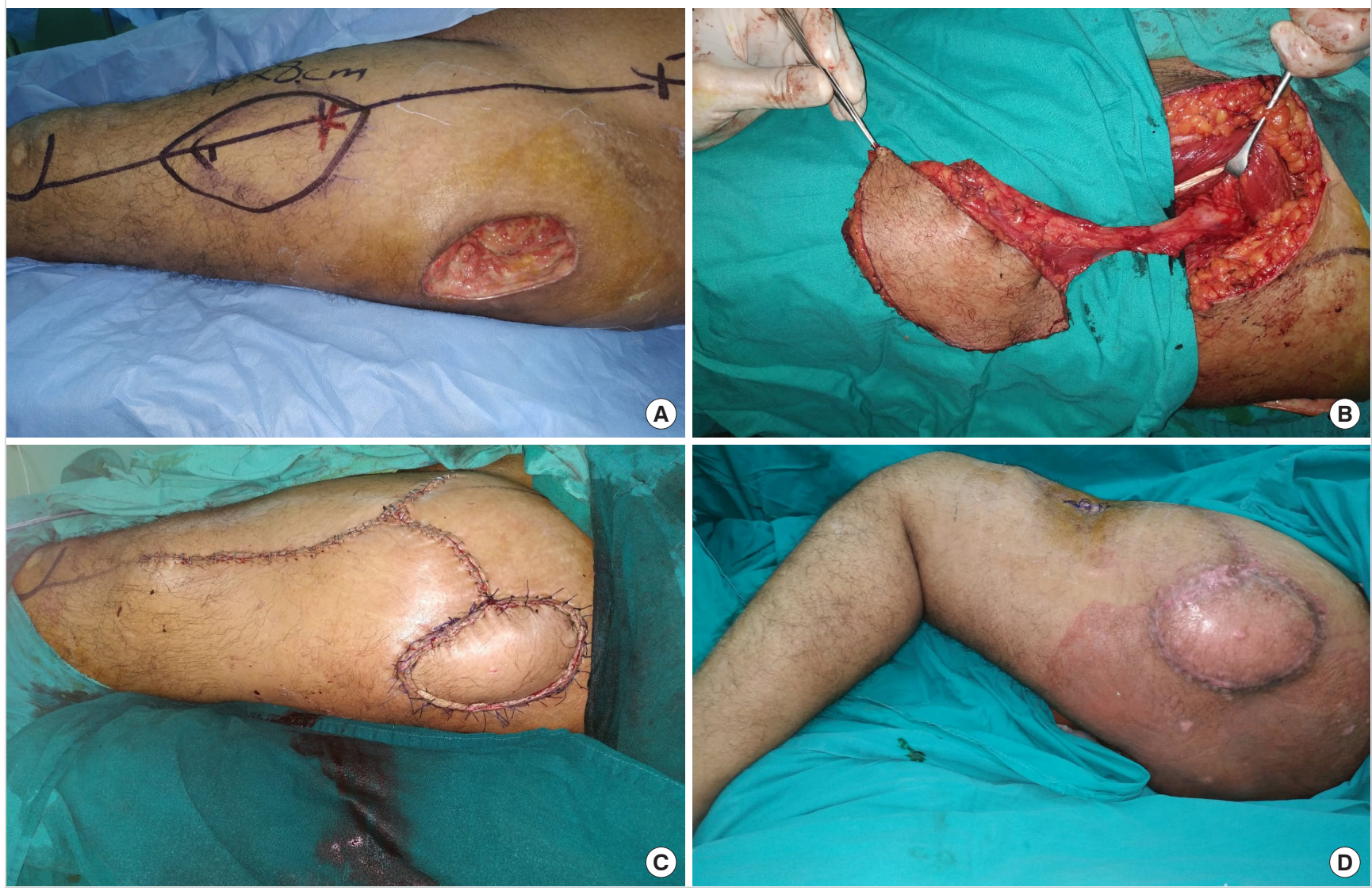

age follow-up period was 6.9 months (range, $2-14$ months) and there was no recurrence during follow-up (Fig. 2D).

\section{DISCUSSION}

Chronic trochanteric ulcers constitute a major cause of morbidity in paraplegic patients as result of greater motion over the trochanter, especially when the lateral decubitus position is assumed. For advanced-stage trochanteric pressure sores, the mainstay of management is surgery, which involves wound debridement followed by soft tissue coverage.

Until recently, the TFL flap has been considered the gold standard technique for the management of trochanteric pressure sores. However, its original design has many disadvantages; for instance, the most poorly vascularized distal portion is usually placed into the bed of the sore, along with meaning an associated dog-ear deformity, which led surgeons to propose new modifications [13-16]. Despite these successful alternatives, some problems remain, including limited flap size that would provide insufficient tissue for a large defect [9], a lack of adequate muscle bulk to fill up the potential dead space, and limited flap mobility with excessive tension during closure.

We suggest that the pedicled ALT myocutaneous flap can overcome the previously mentioned shortcomings, and should therefore be considered as an excellent choice for reconstructing trochanteric pressure sores. However, few studies have reported the use of the pedicled ALT flap for this purpose. In 2007, Chang [8] described an alternative method for trochanteric pressure sore reconstruction by utilizing an island pedicled ALT musculocutaneous flap in a male paraplegic patient. In 2008, Tzeng et al. [10] reported the use of an ALT fasciocutaneous flap for trochanteric pressure sore coverage in a limited sample of four patients. In 2011, Wang et al. [9] demonstrated a modified design of the pedicled ALT myocutaneous flap for trochanteric pressure sore reconstruction in a large case series of 21 patients, which provided a reliable method for easy flap harvesting.

In our study, we consecutively used proximally-based pedicled 


\section{Fig. 3. ALT flap with the open tunnel technique}

(A) Preoperative appearance of a right trochanteric pressure sore. (B) Design of a pedicled anterolateral thigh (ALT) myocutaneous flap measuring $14 \times 8 \mathrm{~cm}$; the flap skin paddle was designed around the most distal detected perforator (red dot), which should be found in the proximal third of the flap. (C) Passage of the flap using the open tunnel technique. (D) Immediate postoperative view of the ALT flap.
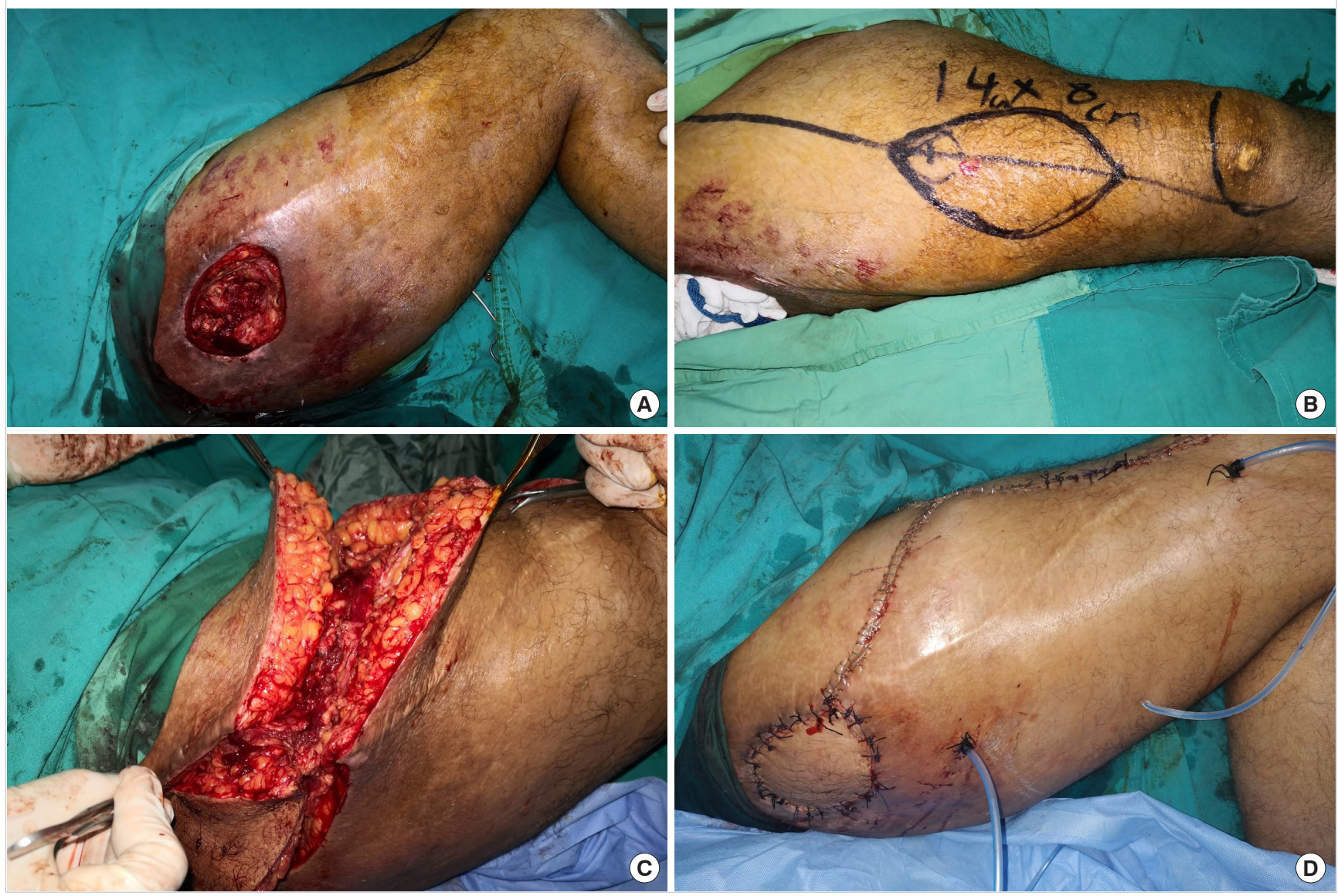

ALT flaps to cover 11 trochanteric pressure sores in 10 patients. To ensure excellent reconstructive outcomes with respect to coverage of the trochanteric region with pedicled ALT flaps, several technical aspects for successful transposition of the pedicled ALT flap should be considered to extend the flap's reach [17]. These technical points include proper flap design regarding perforator selection and the choice of the tunneling technique.

Regarding flap design, Kimata et al. [18] advised using the most distal perforator to attain the longest possible pedicle. Therefore, Wang et al. [9] advocated marking the entire skin island of the ALT flap over the distal half of the thigh, which mainly contains small musculocutaneous perforators, to obtain a longer pedicle for coverage of the trochanteric region. However, in our study, the desired flap size was designed around the most distal perforator detected using Doppler ultrasonography, which should be placed eccentrically in the proximal third of the skin paddle. All flaps could be raised successfully in our patients with a long enough vascular pedicle to transpose the flap easily without restricting the arc of rotation to achieve tension-free closure over the trochanteric ulcer site.

After the completion of flap design, the ALT flap was raised as a pedicled myocutaneous flap to cover trochanteric pressure sores, as suggested by several authors $[8,9]$. This method allows an easy, fast, and safe flap harvesting procedure as it does not require perforator skeletonization [9]. The muscle portion delivers robust vascularity to treat an infected wound bed; additionally, it provides a bulky padding to fill the potential dead spaces, thereby limiting the formation of seroma and hematoma, which might compromise flap survival.

With respect to the tunneling technique, it is crucial to keep the vascular pedicle in an absolutely tension-free position when advancing the flap into the defect. Therefore, various tunneling methods have been described to effectively maximize the vascular pedicle length and to place the pedicle under minimal tension [17]. In previous reports of using the ALT myocutaneous flap for trochanteric region reconstruction, the authors [8-11] were able to pass all flaps either through a lateral subcutaneous 
tunnel or via a pathway beneath the TFL in order to reach the trochanteric wound. In our case series, it was only possible to perform subcutaneous tunneling of the flap to reach the recipient site safely in five patients. In the other six patients, the open tunnel technique was used, with a division of the skin bridge between the recipient and donor sites in order to avoid excessive compression of the vascular pedicle. We realize that the lateral subcutaneous tunnel is considered the easiest route for passing the flap to the defect in underweight or normal-weight patients who have relatively minimal subcutaneous fat in their thighs. Moreover, we believe that this tunneling technique is a more acceptable transfer method in older patients with potentially atrophic thighs or in Asian populations with inherently thin subcutaneous fat in their thighs, as reported in previous studies [811]. However, the open tunnel technique has been proposed as a safer route, especially in overweight or obese patients with a significant amount of subcutaneous fat in their thighs. The decision regarding the choice of tunneling method is made intraoperatively. The skin portion of the flap has a thick fatty layer, which makes the flap bulky. In addition, these patients have a significantly thick lateral subcutaneous tunnel, making it relatively difficult from a technical standpoint to pass a bulky flap without placing excessive tension on the flap vascular pedicle. Consequently, we believe that an open tunnel passageway is necessary in these patients to minimize the risk of pedicle compression, to maximize the flap reach, and to obtain tension-free closure. Additionally, there is a possible risk of avulsion of the vascular pedicle during flap transfer due to possible excessive tension.

Furthermore, we agree with Kua et al. [19] and Wang et al. [9], who recommend incising part of the anterior border of the vastus lateralis muscle in order to make a trough, for easy insetting of the flap pedicle. The amount of muscle to be excised depends on the situation. This maneuver allows a more direct path for the pedicle and reduces tension from pedicle stretching.

The pedicled ALT myocutaneous flap achieves excellent reconstructive outcomes for reconstructing trochanteric pressure sores; therefore, we suggest that the pedicled ALT flap should be the first option for trochanteric ulcer reconstruction. It provides a large, durable skin island and a bulky muscle for soft tissue defect coverage and to fill up the ulcer dead space [20], respectively. However, several strategies in flap design and tunneling should be highlighted to maximize the flap's reach. Incorporating the most distal perforator into the designed skin paddle is suggested to obtain an adequate pedicle length. Although tunneling of the flap through a lateral subcutaneous tunnel is advised in thin patients to minimize scarring, it is a risky approach in overweight or obese patients with a significant amount of subcutaneous fat in their thighs. For this reason, the open tunnel technique is advocated for safer flap transfer to avoid the risk of pedicle compression.

The pedicled ALT flap is a safe and reliable option for reconstructing trochanteric pressure sores. Appropriate flap design and a suitable choice of the tunneling method are essential for successful transposition of the flap for reconstruction of the trochanteric region.

This is a relatively small retrospective case series with the corresponding inherent limitations; therefore, more cases are needed to highlight the differences between the two tunneling methods and to clarify when to choose one technique over the other. We also need to identify the factors that affect the selection of the open technique and to clarify whether this choice is related only to patients' body habitus or body mass index, or other factors such as pedicle length, which may be shorter in overweight patients.

\section{NOTES}

\section{Conflict of interest}

No potential conflict of interest relevant to this article was reported.

\section{Ethical approval}

The study was approved by the Institutional Review Board of Qena Faculty of Medicine (IRB No. SVU-MED-PLS-0134201087) and performed in accordance with the principles of the Declaration of Helsinki. Written informed consent was obtained.

\section{Patient consent}

The patients provided written informed consent for the publication and the use of their images.

\section{ORCID}

Mahmoud A. Hifny https://orcid.org/0000-0001-8118-6166

\section{REFERENCES}

1. Disa JJ, Carlton JM, Goldberg NH. Efficacy of operative cure in pressure sore patients. Plast Reconstr Surg 1992;89: 272-8.

2. Nahai F, Silverton JS, Hill HL, et al. The tensor fascia lata musculocutaneous flap. Ann Plast Surg 1978;1:372-9.

3. Neligan PC, Lannon DA. Versatility of the pedicled anterolateral thigh flap. Clin Plast Surg 2010;37:677-81.

4. Ng RW, Chan JY, Mok V, et al. Clinical use of a pedicled an- 
terolateral thigh flap. J Plast Reconstr Aesthet Surg 2008;61: 158-64.

5. Zelken JA, AlDeek NF, Hsu CC, et al. Algorithmic approach to lower abdominal, perineal, and groin reconstruction using anterolateral thigh flaps. Microsurgery 2016;36:104-14.

6. Friji MT, Suri MP, Shankhdhar VK, et al. Pedicled anterolateral thigh flap: a versatile flap for difficult regional soft tissue reconstruction. Ann Plast Surg 2010;64:458-61.

7. Hsu H, Chien SH, Wang CH, et al. Expanding the applications of the pedicled anterolateral thigh and vastus lateralis myocutaneous flaps. Ann Plast Surg 2012;69:643-9.

8. Chang SH. Anterolateral thigh island pedicled flap in trochanteric pressure sore reconstruction. J Plast Reconstr Aesthet Surg 2007;60:1074-5.

9. Wang CH, Chen SY, Fu JP, et al. Reconstruction of trochanteric pressure sores with pedicled anterolateral thigh myocutaneous flaps. J Plast Reconstr Aesthet Surg 2011;64:671-6.

10. Tzeng YS, Yu CC, Chou TD, et al. Proximal pedicled anterolateral thigh flap for reconstruction of trochanteric defect. Ann Plast Surg 2008;61:79-82.

11. Saint-Cyr M, Uflacker A. Pedicled anterolateral thigh flap for complex trochanteric pressure sore reconstruction. Plast Reconstr Surg 2012;129:397e-399e.

12. Saint-Cyr M, Oni G, Lee M, et al. Simple approach to harvest of the anterolateral thigh flap. Plast Reconstr Surg 2012; 129:207-11.

13. Paletta CE, Freedman B, Shehadi SI. The VY tensor fasciae latae musculocutaneous flap. Plast Reconstr Surg 1989;83:
852-8.

14. Siddiqui A, Wiedrich T, Lewis VL Jr. Tensor fascia lata V-Y retroposition myocutaneous flap: clinical experience. Ann Plast Surg 1993;31:313-7.

15. Lynch SM. The bilobed tensor fascia lata myocutaneous flap. Plast Reconstr Surg 1981;67:796-8.

16. Demirseren ME, Gokrem S, Ozdemir OM, et al. Hatchetshaped tensor fascia lata musculocutaneous flap for the coverage of trochanteric pressure sores: a new modification. Ann Plast Surg 2003;51:419-22.

17. Vijayasekaran A, Gibreel W, Carlsen BT, et al. Maximizing the utility of the pedicled anterolateral thigh flap for locoregional reconstruction: technical pearls and pitfalls. Clin Plast Surg 2017;44:371-84.

18. Kimata Y, Uchiyama K, Ebihara S, et al. Versatility of the free anterolateral thigh flap for reconstruction of head and neck defects. Arch Otolaryngol Head Neck Surg 1997;123:132531.

19. Kua EH, Wong $\mathrm{CH}, \mathrm{Ng} \mathrm{SW}$, et al. The island pedicled anterolateral thigh ( $\mathrm{pALT}$ ) flap via the lateral subcutaneous tunnel for recurrent ischial ulcers. J Plast Reconstr Aesthet Surg 2011;64:e21-3.

20. Hifny MA. Rotation advancement VY posterior thigh flap combined with a biceps femoris muscle flap for reconstruction of a recurrent ischial pressure sore in pediatric patients. Eur J Plast Surg 2020 May 7 [Epub]. https://doi.org/10.1007/ s00238-020-01684-2. 